\title{
Ueber die Entstehung der falschen Knoten der Nabelschnur nebst Bemerkungen iiber die Bulbi und Varicen der Nabelgefässe.
}

\author{
Von \\ Prof. Dr. R. Thoma, \\ Magdebutty.
}

(Mit 9 Abbildungen in Text.)

Bekanntlich stellen sich die falschen Knoten der Nabelschnur dar als knäuel- oder schlingenförmige Verkrümmungen der Gefässe, in deren Umgebung in der Regel etwas reichlichere Anhäufungen von Wharton'scher Sulze nachweisbar sind. An Stelle dieser Verkrümmungen erfahren die Spiralen der Nabelgefässe eine unvermittelt auftretende Störung. Dabei können die Spiralen im Uebrigen durchacus regelmässig verlaufen, oder sie zeigen mancherlei $\Lambda$ bnormitäten. Häufig kommt es vor, dass die Drehungsrichtung der Spiralen der Nabelschnurgefässe oberhalb und unterhalb des falschen Knotens verschieden ist, oberhalb des Knotens rechtsläufig und unterhalb desselben linksläufig oder umgekehrt.

Hyrtl' ${ }^{1}$ ) hat solche falsche Knoten der Nabelschnur in sehr eleganter Weise zur Anschauung gebracht, indem er die mit gefärbten Harzmassen injicirten Nabelschnüre der Corrosion unterwarf. Zweckmässiger ist die Paraffininjection mit nachfolgender Corrosion, welche ich bei einer anderen Gelegenheit zusammen mit Fromherz's verwendet habe. Sie bietet den Vorzug, dass man bei ungleich

1) Hyrtl, Die Bulbi der Placentararterien. Denkschr. d. J. Acad. in Wien. Math.-naturw. Cl. Bd. 29. 1869. - Die Blutgefässe der menschlichen Nachgeburt. Wien 1870.

2) Thoma u. Fromherz, Ueber die lichte Weite der Placentararterien. Arch. f. Entwicklungsmechanik d. Organismen. Bd. 7. 1898. 
Thoma, Ueber die Entstehung der falschen Inoten der Nabelschnur. 37

niedrigerer Temperatur injiciren kann. Für einfacher gebaute falsche Knoten ist auch die Injection mit Alcohol (950 Tralles) zu empfehlen. Die Erklärung der Entstehung der falschen Knoten hat indessen jederzeit grosse Schwierigkeiten bereitet. Sie führt zunächst auf die Frage nach den Ursachen der Spiraldrehung der Nabelschnur, denn die falsche Knotenbildung scheint sich als eine Störung der letzteren darzustellen.

Haller ${ }^{1}$ ) war der Meinung gewesen, dass die Spiraldrehung der Nabelschnur veranlasst werde durch Untersehiede in der Wachsthumsgeschwindigkeit der Nabelgefässe einerseits und der amniotischen Scheide derselben andererseits. Das raschere Längenwachsthum der Nabelschnurgefässe solle diese zwingen, sich in der langsamer wachsenden Amuionscheide in Spiralen zu legen.

Diese Erklärung Haller's beruft sich auf die Thatsache, dass die Länge der Nabelschnurgefässe, wenn man diese entlang ihrem spiraligen Verlanfe misst, grösser ist als die Länge der Nabelschnurscheide, gemessen parallel der Axe der Nabelschnur. Haller macht hier die Voraussetzung, dass die Amnionscheide an der Spiraldrehung nicht theilnehme. Unter dieser Voraussetzung ist seine Beweisführung wenigstens in so weit richtig, als sie ein rascheres Längenwachsthum der Blutgefässe nachzuweisen versucht. Unerklärlich aber bliebe immerhin noch die Regelmässigkeit der Spiralen.

Wenn man dagegen die Annahme macht, dass bei der Spiraldrehung der Nabelschnurgefässe auch die Amnionscheide spiralig aufgedreht wird, so muss man selbstverständlich auch die Länge der letzteren entlang dem Verlaufe der Spiralen messen. In diesem Falle wird sodann die Länge der Amnionscheide genau gleich der Länge der Nabelschnurgefässe. Damit würde die Haller'sche Erklärung ihre Grundlage verlieren und wäre widerlegt.

Ich möehte an dieser Stelle vorläufig noch kein endgültiges Urtheil über die Haller'sche Lehre abgeben. Man bemerkt indessen, dass ihre Voraussetzung, die Amnionscheide nehme an der Spiraldrehung nicht Theil, zugleich auch wiederholte Drehungen des Foetus in der Amnionhöhle mit Bevorzugung einer Drehungsrichtung ausschliesst. Jede zufälliger Weise erfolgende Drehung des Foetus müsste, wenn die Amnionscheide keine Spiraldrehung erfahren soll, in kurzer Zeit durch eine Drehung in entgegengesetzter Richtung wieder ausgeglichen werden.

1) Haller citirt nach Hyrtl a. a. O. 
38 Thoma, Ueber die Entstehung der falsehen Knoten der Nabelschnur.

Nan hat weiterhin die Unterschiede im Kaliber beider Nabelarterien in Verbindung mit den Krümmungen, welche die Nabelgefässe bei ihrem Austritte aus dem Körper und bei ihrem Uebergange auf die Placenta aufweisen, verantwortlich gemacht für die physiologische Spiraldrehung der Nabelschnur [Neugebauer ${ }^{1}$ ), Simpson' $\left.{ }^{2}\right)$ ]. Sind, wie man zugeben darf, beide Nabelarterien von ungleichem Kaliber, so ist es wohl denkbar, dass der Rückstoss des Blutstromes und die ungleiche Spannung der Gefässwandungen im Stande sei, der Nabelschnur und dem Foetus eine langsam zunehmende Drehung zu verleihen. Indessen hat Stutz ${ }^{3}$ ) darauf hingewiesen, dass die Nabelschnur in der ersten Zeit einen gestreckten Verlauf besitzt und der Spiraldrehung entbehrt, obwohl nach den Voraussetzungen von Neugebauer und Simpson bereits in dieser ersten Zeit Spiraldrehungen zu erwarten wären.

Man darf daher nicht ausser Betracht lassen, dass der von den beiden letztgenannten Autoren erwartete Erfolg nicht nothwendiger Weise eintreten muss. Vielmehr könnten die genannten Ungleichheiten der beiden Nabelarterien sehr wohl auch in anderer Weise ihren Ausgleich finden, etwa durch schräge Einpflanzung der Nabelschnur auf dem Nabel und auf der Placenta. Eine solche schräge Einpflanzung der Nabelschnur könnte zur Folge haben, dass die an Kaliber stärkere Arterie einen schwächeren Bogen beschreiben würde beim Uebergang in die Nabelschnur. Dann wären die drehenden Wirkungen des Rückstosses in beiden Árterien möglicher Weise der Grösse nach gleich und der Richtung nach entgegengesetzt und würden sich demgemäss gegenseitig aufheben.

Es bleibt somit auch das Urtheil über die Anschaungen von Neugebauer und Simpson vorläufig unentschieden. Damit aber crgiebt sich zugleich die Frage, ob noch andere Umstände auf die Beweglichkeit des Foetus in der Amnionhöhle Einfluss haben können.

Mit der Entwicklung der Nabelschnur und mit der wachsenden Grösse des Foetus tritt nothwendiger Weise früher oder später ein Zeitpunkt ein, in welchem der Foetus vermöge der Länge der Nabelschnur zwar zu ausgiebigen Bewegungen befähigt ist, jedoch in seiner freien Beweglichkeit in der Amnionhöhle einigermaassen

1) Neugebauer, Die Morphologie der menschlichen Nabelschnur. Breslau 1858.

2) Simpson, Edinburgh med. Journ. Bd. V. $18599-1860$.

3) Stutz, Dieses Archiv. Bd. 13. 1878. 
Thoma, Ueber die Entstehung der falschen Knoten der Nabelschnur. 39

beschränkt wird durch seine Berührungen mit den Wandungen dieser Höhle. Es ist dann mit Sicherheit vorauszusehen, dass er gelegentlich in Folge von Bewegungen seiner Extremitäten und in Folge von Aenderungen der Körperhaltung der Mutter Drehungen ausführen wird, welche zu einer Spiraldrehung der Nabelschnur führen.

Weiterhin muss man annehmen, dass die Gestaltung der Eihöhle, die Haltung des Fötus, sowie die Unterschiede in dem specifischen Gewichte beider Körperhälften des Fötus Drehungen in der einen Richtung mehr begünstigen, als in der entgegengesetzten Richtung. Sicherlich wird nun die Drehungsrichtung gelegentlich umkehren. Wenn aber die Drehung in der einen Richtung bebegünstigt wird, muss das Gesammtergebniss sich als eine wiederholte Drehung der Nabelschnur darstellen. Dass sodann die Mehrzahl der Nabelschnüre eine linksläufige Spirale aufweisen, kann schliesslich nicht auffallen, da die für die Drehungsrichtung maassgebenden Factoren, die Asymmetrien des mütterlichen und des fötalen Körpers, die asymmetrische Lage des Fötus in der Amnionhöhle und das ungleiche specifische Gewicht beider Körperhälften des Kindes sich ungeachtet zahlreicher individueller Verschiedenheiten einer bestimmten Norm nähren. Nan muss dann annehmen, dass diese als Norm ${ }^{1}$ ) zu bezeichnenden Verhältnisse die Linksdrehung der Nabelschnur begünstigen.

Diese Auffassung der physiologischen Verhältnisse nähert sich derjenigen ron Velpeau, wenigstens in den wesentlichsten Punkten. Wenn man nun annimmt, dass die normale Spiraldrehung der Nabelschnur Folge ist von Drehungen des fötalen Körpers, so erkennt man, dass dabei auch jeder einzeine, in der Nabelschnur gelegene Gefässstamm eine entsprechende Spiraldrehung erfahren muss. Diese aber führt nothwendiger Weise zu inneren Torsionsspannungen in den Gefässwandungen, in der Wharton'schen Sulze und in der Amnionseheide. So lange diese inneren Spannungen bestehen, kamn durch Rückdrehung der Spirale der frühere Zustand wieder bergestellt werden. Zweifellos werden jedoch in der lebenden Nabelschnur diese inneren Torsionsspannungen der Gewebe

1) Ueber den Begriff der Norm and seine Begründung vergl. Thoma, Untersuchungen über die Grösse und das Gewicht der anatomischen Bestandtheile des menschlichen Körpers im gesunden und im kranken Zustande. Leipzig 1882. 
40 Thoma, Ueber die Entstehung der falschen Knoten der Nabelschnur.

allmälig durch Wachsthumsvorgänge ausgeglichen. Soweit dies geschieht, ist dann die Spiraldrehung nicht wieder ohne Weiteres aufzuheben, weil bei dem Versuche, die Spirale zurückzudrehen, wieder von Neuem Torsionsspannungen in den Geweben der Nabelschnur entstehen, welche das Zurückdrehen der Spirale hindern.

Nach diesen Gesichtspunkten lässt sich sehr wohl die Regelmässigkeit der normalen Spiraldrehung der Nabelschnur erk]ären, allein es fragt sich nun, ob auch die Anomalien der Torsion der Nabelschnur und die Bildung der falschen Knoten sich aus denselben ableiten lassen.

Zunächst ist es klar, dass die stenosirenden und occludirenden. Torsionen der Nabelschnur dringend auf dieselbe Erklärung hinweisen. Diese entstehen sogar zweifellos zum Theile erst nach erfolgtem Tode des Fötus, also unter Umständen, unter denen Wachsthumsverschiedenheiten zwischen den Nabelschnurgefässen und der Nabelschnurscheide und Rückwirkungen des Blutstromes auf das Wachstbum der Gefässwand auszuschliessen sind. Diese stenosirenden und occludirenden Torsionen können auch, soweit sie nach dem Tode des Fötus zu Stande gekommen sind, wieder mehr oder weniger vollkommen zurückgedreht und beseitigt werden, wenn auch die Wharton'sche Sulze durch die schnürende Wirkung der vielfältigen Torsion Verschiebungen erfahren hat, welche sich schliesslich mit den Erscheinungen der Aufquellung des abgestorbenen Gewebes verknüpfen.

Es ist weiterhin verständlich, dass schwach gedrehte und nahezu gestreckt verlaufende Nabelschnüre vorkommen, wenn durch besondere Verhältnisse die Drehung des Fötus in keiner Richtung bevorzugt wird, sodass die Drehungsrichtung häufig umkehrt. In anderen Fällen mag es vorkommen, dass die bevorzugte Drehungsrichtung des Fötus sich in den späteren Schwangerschaftsmonaten, unter der Mitwirkung besonderer Umstände, umkehrt. Wenn dann die neu auftretenden, im umgekehrten Sinne gerichteten Torsionsspannungen der Nabelschnur in dem Maassstabe, wie sie entstehen, durch Wachsthumsvorgänge ihren Ausgleich finden, können sich wiederum schwach gedrehte oder nahezu gestreckt verlaufende Nabelstränge ergeben. Es könnte sich sogar ereignen, dass auf diesem Wege die Richtung der Windungen der Nabelschnur umgekehrt würde. Doch lässt sich solehes selbstverständlich nur nachweisen, wenn die Umkehr der Windungsrichtung sich auf einen Theil der Nabelschnur beschränkt. Solche Nabelschnüre, welche 
Thoma, Ueber die Entstehung der falschen Knoten der Nabelschnur. 41

stellenweise rechtsläufige, stellenweise linksläufige Spiralen aufweisen, kommen nicht allzu selten vor. Doch wird man in solchen Fällen zugleich annehmen müssen, dass ein Theil der Nabelschnur, durch irgend welche Umstände, vielleicht durch Verschlingung um eine Extremität, gegen die Wirkung der Rückdrehung geschützt wurde. Alle diese Umformungen aber setzen zweifellos voraus, dass sich die Drehungen des fötalen Körpers nicht allzu häufig wiederholen, damit die Gewebe der Nabelschnur Zeit haben, die Torsionsspannungen durch entsprechende gewebliche Vorgänge auszugleichen.

Bei dem Zurückdrehen einer bereits fertig gebildeten. Spirale der Nabelschnur kann sich jedoch noch eine andere Störung einstellen, indem durch die neu entstehenden Torsionsspannungen die Gefässe stellenweise auseinandergedrängt und in Knäuel oder Schlingen gelegt werden, welche man als falsche Knoten zu bezeichnen pflegt. Hyrtl hat seiner Zeit die Anschaumgen ron Velpeau, denen zu Folge die physiologische Torsion der Nabelschnur Folge sein soll von Drehungen des fötalen Körpers, hauptsächlich aus dem Grunde zurückgewiesen, weil. es ihm unmöglich erschien, auch dic falseben Knoten der Nabelschnur durch irgendwelche Drehungen des Fötus oder der Nabelschnur zu erklären. In dieser Beziehung konnten ihn auch die vergeblichen Versuche Simpson's nur bestärken. Doch ist es sehr leicht und einfach, die falschen Knoten der Nabelschnur als Torsionsanomalien zu erweisen.

Man verschaffe sich ein Stück guter, gedrehter Hanfschnur, welche noch keine Abnützung aufweist. Diese Hanfschnur soll aus drei, gleichfalls scharf gedrehten Strängen zusammengedreht sein. Die Enden der Hanfsehnur kann man, wenn man will, mit je einem Knoten versehen oder einzeln mit dünnem Zwirn umwickeln, damit sie nicht auffasern. Nothwendig ist dieses indessen nicht.

Versucht man nunmehr die Mitte der Hanfschnur zurückzudrehen, so leistet das Material einen gewissen Widerstand, weil innere Torsionsspannungen entstehen, welche sich dem Rückdrehen entgegenstellen. Dreht man stärker, so gelingt indessen letzteres. Die erzeugten starken Torsionsspannungen in den drei Strängen, aus denen die Hanfschnur besteht, finden zugleich wenigstens theilweise einen Ausgleich, indem nun die einzelnen Stränge sich in Schlingen 
42 Thoma, Ceber die Entstehung der falschen Knoten der Nabelschnur.

legen. Man erhält dabei eine Bildung (Fig. 1), welche einem durch Corrosion klar gelegten falschen Knoten der Nabelschnurgefässe (Fig. 2) durchaus entspricht. Indessen zeigt sich in dem hier abgebildeten falschen Nabelschnurknoten die Spirale der Nabelschnurgefässe oberhalb des Knotens nach rechts gedreht, während nnterhalb des Knotens eine links gewundene Spirale vorliegt.

Diese auch in anderen Fällen nicht selten vorkommende Besonderheit des hier gezeichneten Nabelschnurknotens ist mit der

Fig. 1.

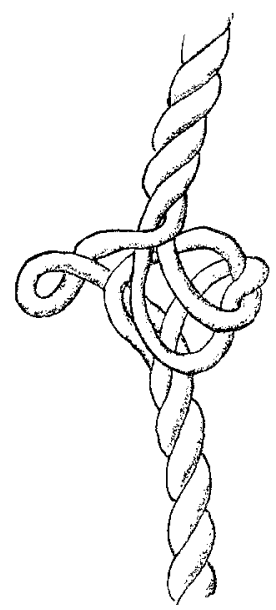

Falscher: Knoten in einer Hanfschnur, durch Zurückdrehen derselben erzeugt.
Fig. 2.

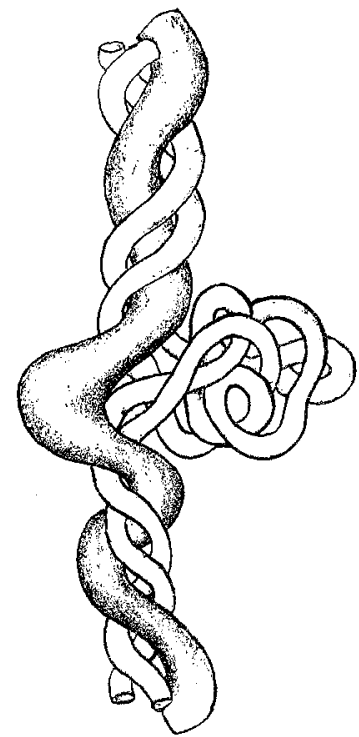

Falscher linoten der Nabelschnur nach Hyrtl. Verklejnert. Arterien hell, Vene dunkel gezeichnet.

Hanfschnur nicht nachzuahmen ohne Anwendung überlegener, schwerer Gewalt. Leichter gelingt dies mit Gummischläuchen. Man lege drei schwarze Gummischläuche zu einem Bündel zusammen und umschnüre das eine Ende dieses Bündels mit einer festen Ligatur. Die drei freien Enden der Schläuche schiebt man sodannn durch drei Löcher, welche in ein kleines Brettchen b (Fig. 3) gebohrt sind, und rückt das Brettehen bis in die Nähe der Ligatur a. Wenn man nun die Ligatur bei a mit der einen Hand festhält, kann man mit der anderen Hand das Brettchen so drehen, dass sich die drei Gummischläuche in regelmässigen Spiralwindungen 
Thoma, Ueber die Entstehung der falschen Knoten der Nabelschnur. 43

zusammenlegen. Schliesslich legt man oberhalb des Brettchens eine zweite Ligatur um das Bündel der Gummischlänche (Fig. 4).

Giebt man nun die beiden Enden des Schlauchbündels frei, indem man zugleich das Brettchen entfernt, so gehen zwar einige Windungen der Spirale wieder verloren, die meisten Spiralen bleiben jedoch bestehen und man erhält ein Gebilde, welches einer Nabelschnur nicht unähnlich sieht. Doch ist es bei diesen Vorbereitungen wünschenswerth, dass die drei Löcher in dem Brettchen genügend weit seien, damit sich die Schläuche in denselben während der Bildung der Spirale leicht drehen können. Auch sollten die drei

Fig. 3 .

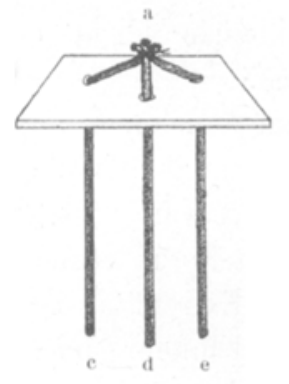

Fig. 4.

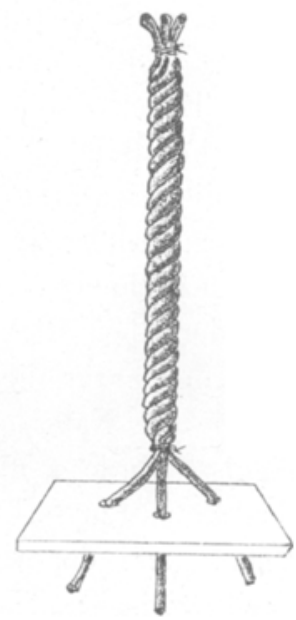

Gummischläuche und durchbohrtes Spiralig zusammengedrehtes Bündel Brettchen. Erklärung im Text. yon Gummischläuchen.

Löcher in dem Brettchen gleich weit von einander abstehen, indem sie etwa die Ecken eines gleichschenkligen Dreiecks einnehmén.

Mit diesem Bündel spiralig gedrehter Gummischläuche kann man ohne allzugrosse Schwierigkeit die Umkehr der Spiralwindungen der Nabelschnur nachbilden, wenn sich auch dabei erhebliche Torsionsspannungen einstellen, welche verhindern, dass die Umkehr der Spiralwindungen bestehen bleibt, wenn man die Enden des Schlauchbündels wieder freigiebt. Es stellt sich in diesem Falle die ursprüngliche Spiralwindung des Schlauchbündels wieder ein. Man ist in der That nicht im Stande, mit unbelebtem Materiale diejenigen Umformungen der Nabelschnur in befriedigender Weise 
44 Thoma, Leber die Entstehung der falschen Knoten der Vabelschnur.

zu wiederholen, bei welchen Vorgänge der Neubildung, Umbildung und Rückbildung von Geweben in ausgiebiger Weise mitwirken. Wohl aber gewinnt man eine Anschauung über die bei der Umkehr der Spiralwindungen sich vollyichenden Vorgänge.

Dagegen gelingt es sehr gut, mit dem spiraliggedrehten Bündel von Gummischläuchen die falschen Knoten zu erzeugen, auch diejenigen, von welchen weiterhin die Rede sein wird. Es ist dies zu erwäbnen, um dem Einwurfe zu begegnon, dass der scharfe Drall der Hanfschnur etwa die Beweiskraft der Versuche zu beeinträchtigen im Stande sei. Die Hanfschnur giebt nur ein bequem zu behandelndes Material, weil in demselben keine inneren Torsionsspannungen mehr bestehen, welche in dem spiralig zusammengedrehten Bündel der Gummischläuche nicht völlig fehlen. Die Hanfsehnur dreht sich nicht auf, wenn ihre Enden durchschnitten werden, während das spiralig zusammengedrehte Bündel der Gummischläuche leicht auseinander weicht, wenn die Ligaturen gelöst werden.

Nach diesen Erörterungen würde man annehmen könmen, dass die in Fig. 2 gezeichnete Nabelschnur ursprünglich in ihrer ganzen Länge nach derselben Richtung gewunden war. Später düriten sich die Bedingungen für die Drehungen des Fötus geändert haben. Vielleicht wurde die Nabelschnur um eine Extremität geschlungen. Dieses Ereigniss würde genügen, um die Umkehr der Drehungsrichtung des Fötus zu erklären. Zugleich würde es zur Folge haben können, dass der von dem Nabel des Fötus zu der Umschlingungstelle ziehende Theil der Nabelschnur die Drehungen des Fötus mitmachte, und somit in seinen Torsionsverhältnissen nicht weiter verändert wurde. Um so stärker musste selbstverständlich die Umkehr der Drehungsrichtung des Fötus auf den von der Umschlingungsstelle zu der Placenta ziehenden Theil der Nabelschnur wirken. In diesem Theile konnte mit der Zeit die ursprüngliche Drehungsrichtung der Spirale unter der Wirkung der neu entstandenen Torsionsspannungen umgebaut werden.

An der Stelle jedoch, an welcher die linksgedrehte Spirale in die rechtsgedrehte Spirale übergeht, ist offenbar ein kritischer Punkt gegeben, an welchem sich sehr starke Torsionsspannungen entwickeln mussten. Hier wichen die drei Gefässe der Nabelschnur auseinander und bildeten die Schlingen eines falschen Knotens, wodureh ein beträchtlicher Theil der Torsionsspannungen aufgehoben wurde. 
Thoma, Ueber die Entstehung der falschen Knoten der Nabelschnur. 45

Ausserdem findet man hier (Fig. 2) an der Umbilicalvene eine varicöse Erweiterung. Es scheint mir wahrscheinlich, dass diese ihre Entstehung gleichfalls den genannten Torsionsspannungen verdankte, indem diese den Zusammenhang der Gewebselemente der Venenwand lockerten and damit eine passive, durch den Blutdruck bewirkte Erweiterung der Venenlichtung vorbereiteten. Verfertigt man sich das Modell einer spiralig gedrehten Nabelschnur aus zwei engeren dickwandigen und einem weiteren dünnwandigen, schwarzen Gummischlauche und versucht nun an diesem durch Aufdrehen der Spirale einen falschen Knoten zu erzeugen, so entstehen sehr leicht Knickungen des dünnwandigen Schlauches, während die beiden dickwandigen, engeren Schläuche sich in Schlingen legen. Solche durch die Torsionsspannungen erzengten Knickungen namentlich dürften regelmässig in der lebenden Gefässwand zu geringen Trennungen des Zusammenhanges der Gewebselemente und damit zu Erweiterungen der Gefässlichtung Veranlassung geben. Sie erklären somit, wie es scheint, die varicöse Erweiterung und theilweise auch den Mangel der Schingenbildung an der Vene in Fig. 2.

Aehnliche Veränderungen findet man gelegentlich auch an den Arterien des Nabelstranges und der Placenta in Form der sogenannten Bulbi. Diese stellen sich als kleine Aneurysmen dar, die zumeist multipel auftreten. Häufig begleiten sie Torsionsanomalieen anderer Art. Doch ist es klar, dass auch bei der Entwicklung der physiologischen Nabelschnurspiralen in den Wandungen der Blutgefässe gelegentlich so starke Spannungen in irgend welcher Richtung auftreten können, dass die Gewebselemente der Gefässwand über die Elasticitätsgrenze gedehnt werden. Ich denke dabei sowohl an kleinste Zerreissungen der Gewebselemente, welche nur mikroskopisch nachweisbar sein würden, als an dauernde Verlängerungen und Verzerrungen der Gewebselemente. Indem diese eintreten, findet ein Theil der sie veranlassenden Spannungen seinen Ausgleich. Es ist desshalb nicht nothwendig, dass neben den sich anschliessenden umschriebenen Gefässerweiterungen auch falsche Knotenbildungen auftreten. Doch werden beide häufig vereingt gefunden.

Diese Erörterung der besonderen Verhältnisse der Fig. 2 kann eine concrete Vorstellung bezüglich der Entwicklungsgeschichte eines falschen Knotens geben. Ob sie in allen Einzelheiten zutrifft, werden weitere Untersuchungen zu entscheiden haben. Für den Augenblick obliegt mir indessen nur die Beweisführung dafür, dass man durch 
46 Thoma, Ueber die Entstehung der falschen Knoten der Nabelschnur.

Aufdrehung von Hanfschnüren die Besonderheiten der Form der falschen Knotenbildung im Nabelstrang genau nachahmen kann.

Nun bemerkt man jedoch, dass in Fig. 1 die drei Stränge der aufgedrehten Hanfschnur an der Aufdrehungsstelle ungefähr eine gleiche Anzahl von Schlingen werfen. Dagegen ist in Fig. 2 zwar die Zahl der von beiden Arterien geworfenen Schlingen annähernd gleich gross, doch wirft die Vene nur eine einzige Bucht.

Versuche an der oben beschriebenen Hanfschnur zeigen, dass man dies nachahmen kann, wenn man einige Schlingen des einen

Fig. 5 .

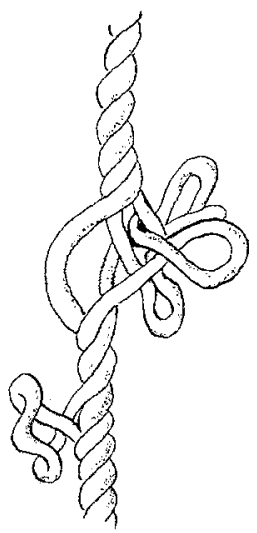

Falscher Knoten in einer Hanfschnur.
Fig. 6.

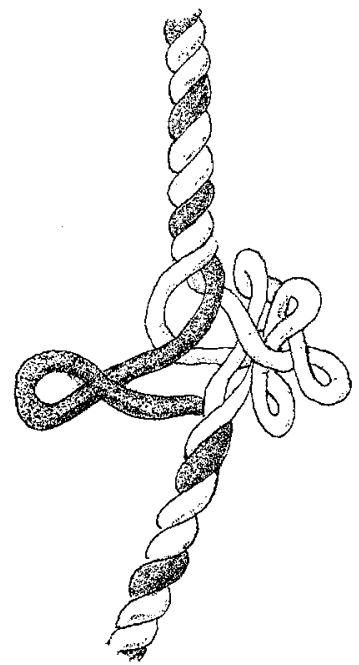

Falscher Knoten in einer Hanfschnur, in welcher ein Faden durch dunkelfarbige Wolle ersetzt ist.

Stranges an der Spirale nach oben oder unten schiebt, indem man somit die Torsionsspannungen in diesem einen Strange an einer anderen Stelle der Schnur zum Ausgleich bringt. Es ergibt sich dann etwa das Verhalten der Fig. 5. Nach den von Hyrtl abgebildeten Corrosionspräparaten zu schliessen, kommen solche Ausgleiche der Torsionsspannungen an verschiedenen Stellen der Nabelschnur vor. In dem Falle der Fig. 2 scheinen sie jedoch nicht massgebend gewesen zu sein. Das Verbalten der Vene ist hier vielmehr bestimmt durch den Umstand, dass die Wand der Vene ungleich schwächer ist und daher durch die gleiche Zahl von Drehungen eine geringere Torsionsspannung erfährt als die Arterien- 
T'homa, Ueber die Entstehung der falsehen Knoten der Nabelschnur. 47 wand. Ausserdem ist wohl auch, wie oben berührt, ein Theil der Torsionsspannungen in derVenenwand erschöpft worden bei der Ueberdehnung der Gewebe, welche der varicösen Erweiterung voranging.

Fig. 7.

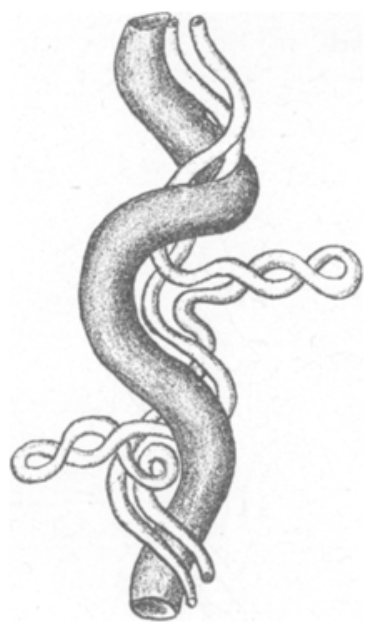

Falscher Knoten der Nabelschnur nach Hyrtl. Verkleinert.

Fig, 8.

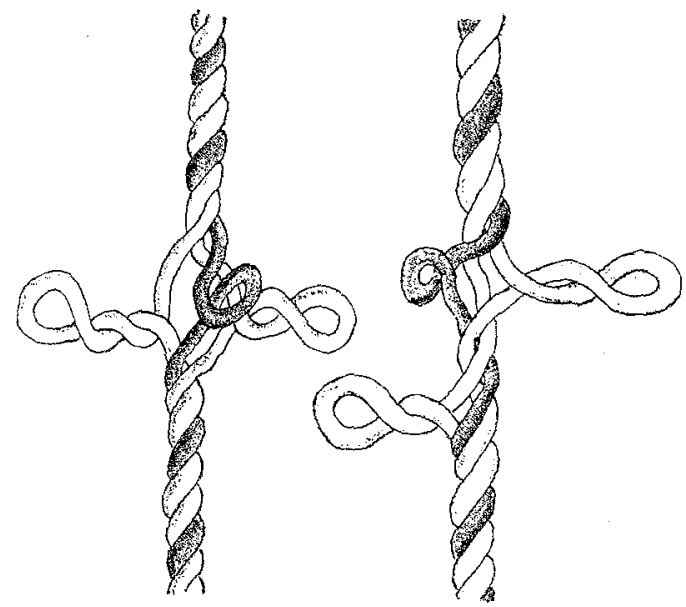

Falsche Knoten in Hanfschnüren, in welchen je ein Hanffaden durch dunkelfarbige Wolle ersetzt ist.

Man kann das Verhalten der Venenwand dadurch nachahmen, dass man aus einem neuen Stücke Hanfschnur vorsichtig einen der scharf gedrehten Stränge herausnimmt und an seine Stelle einen 
48 Thoma, Ueber die Entstehung der falschen Knoten der Nabelschnur.

weichen, etwa dunkelfarbigen Wollefaden einzieht. Macht man nunmehr einen Versuch der Aufdrehung, so legen sich die beiden übrig gebliebenen Hanfstränge in eine viel grössere Zahl von Schlingen als der Wollefaden. Zu Fig. 6 ist die Schnur zugleich etwas öfter zurückgedreht, so dass die Zahl der von Hanfsträngen geschlagenen. Schlingen grösser ist als in Fig. 1. Es wird dabei abgesehen von

F'ig. 9.

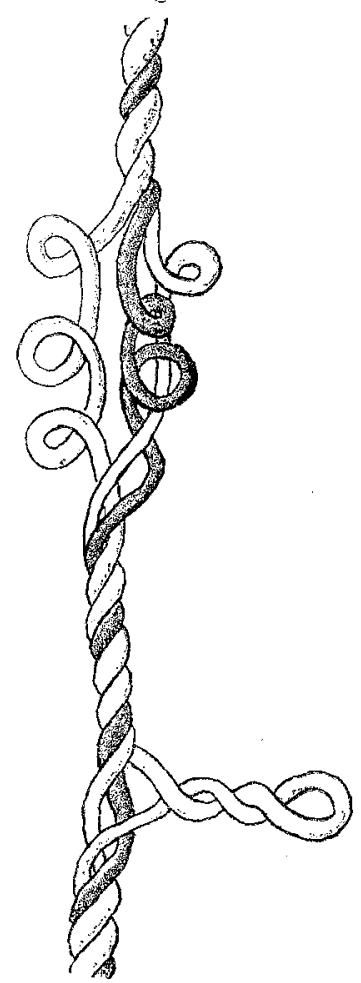

Falsche Knoten in einer Hanfschnur, in welcher ein Hanffaden durch Wolle ersetzt isl.

der varicösen Erweiterung eine ziemlich weitgehende Uebereinstimmung mit dem falschen Nabelschnurknoten der Fig. 2 erreicht.

Durch weitere Versuche kann man mit den gleichen Hülfsmittein leicht alle die sonderbaren Formen von falschen Knoten der Nabelschnur nachbilden, welche in Hyrtl's grossem Werke enthalten sind. In diesem Sinne erscheinen mir vergleichbar die Fig. 7 und Fig. 8, wäbrend die Fig. 9 die eigenartigen Schlingen wiedergibt, welche in den Hyrtl'schen Präparaten der Nabelschnur häufig wiederkehren. Ausführlichere Beschreibungen dieser Figuren dürften 
Thoma, Ueber die Entstehung der falschen Knoten der Nabelschnur. 49

kaum nothwendig sein. Die hier mitgetheilten Versuche erweisen zur Genüge, dass die falschen Knoten der Nabelschnur entstehen durch ein Zurückdrehen der bereits ausgebildeten Spiralen ihrer Blutgefässe.

Bei diesen Versuchen zeigt sich zugleich, dass die Kraft, welche zu einem Zurückdrehen der Spiralen einer Hanfschnur erforderiich wird, zu Anfang eine recht beträchtliche ist. Ueberträgt man dieses Ergebniss auf die drei Blutgefässe der Nabelschnur, welche zudem noch durch die Wharton'sche Sulze untereinander zusammengehalten werden, so bleibt kein Zweifel darüber, dass eine vorangehende Drehung der ganzen Masse des fötalen Körpers nöthig ist, um solche Wirkungen hervorzurufen.

Dagegen genügt eine verhältnissmässig geringere Kraft, um die bereits auseinander gedrängten Spiralen einer Hanfschnur in Sehlingen zu legen und jede weitere Verdrehung der Hanfschnur in gleicher Richtung erzeugt entweder Spiralkrümmungen an diesen Schlingen (Fig. 8 und Fig. 9) oder vermehrt, wenn man das Zustandekommen von Spiralkrümmungen an den Schlingen behindert, die Zahl der Schlingen (Fig. 5 und Fig. 6). Man kann schliesslich durch weiteres Verdrehen der Schnur ausserordentlich verwickelte falsche Knoten herstellen.

Diese Ergebnisse zeigen, dass die Umkehr der bevorzugten Drehungsriehtung des Fötus, welehe als Ursache für die erste Entstehung von falschen Knoten der Nabelschnur betrachtet werden musste, zugleich im Stande ist, die Zahl und Grösse der den falschen Knoten bildenden Blutgefässschlingen zu vermehren. Der falsehe Knoten der Nabelschnur ist somit eines fortschreitenden Wachsthums fähig.

Im Allgemeînen wird man' sich die Vorstellung zu bilden haben, dass jedenfalls das weitere Wachstbum der falschen Knoten ein ganz langsames und allmähliches ist. Vermuthlich gilt das Gleiche auch für das erste Auseinanderweichen der drei Nabelschnurgefässe, welches die Bildung des falschen Knotens einleitet, und für die Bildung der ersten Gefässsschlingen. Die Versuche an den Hanfschnüren sind zwar geeignet, die mechanischen Beziehungen zwischen der Torsionsspannung und der Schlingenbildung aufzudecken, in der lebenden Nabelschnur dürften sich jedoch die Folgen dieser Beziehungen nur durch langsame Verschiebungen der anatomischen Bestandtheile kundgeben. Es ist unter dieser Voraus- 
50 Thoma, Ueber die Entstehung der falschen Knoten der Nabelschnur.

setzung auch anzunehmen, dass bei der Knotenbildung in der lebenden Nabelschnur immer ein grösserer oder kleinerer Theil der entstehenden Torsionsspannungen nicht durch Schlingenbildung, sondern durch Wachsthumsvorgänge seinen Ausgleich findet.

Bei diesen ausgleichenden Wachsthumsvorgängen werden sich zweifellos auch die histo-mechanischen Beziehungen zwischen den Eigenschaften des Blutstromes und den Vorgängen der Neubildung, Umbildung und Rückbildung der Gewebe der Gefässwand geltend machen, welche ich ${ }^{\mathbf{y}}$ ) an anderen Orten klarzustellen versucht habe. Somit scheint die Bildung eines falschen Knotens noch reichen Stoff für weitere Untersuchungen abgeben zu können. Der wichtigste dabei in Betracht kommende Factor dürite aber durch diese Versuche klargelegt sein.

Das Gesammtergebniss lässt bereits heute keinen Zweifel darüber bestehen, dass sowohl die physiologische Spiralwindung der Nabelschnurgefässe als die pathologischen Torsionsanomalien einschliesslich der falschen Knoten in ihrer Entstehung abhängig sind von Drehungen des fötalen Körpers in der Amnionhöhle. Demgemäss dürfen die früher erwähnten Anschauungen von Haller als widerlegt gelten. Dagegen wäre es wohl möglich, dass der Rückstoss des Blutstromes und die Spannung der Gefässwandungen an den Krümmungen, welche die Gefässe am Nabel und bei dem Uebergang auf die Placenta aufweisen, mitbetheiligt wären an der Bestimmung der vorwiegenden Drehungsrichtung des Fötus. Doch ist es klar, dass dieses Moment nicht geeignet erscheint, die pathologischen Torsionsanomalien zu erklären. Er versagt bei der Erklärung der Umkehr der Richtung der Spiralwindungen und bei der Deutung der falschen Knoten. Man ist daher darauf angewiesen, die Asymmetrien des fötalen und des mütterlichen Körpers. und die Ungleichheit des specifischen Gewichtes der verschiedenen Theile des Fötus im Sinne von Velpeau als diejenigen Factoren zu betrachten, welche den grösseren und massgebenden Einfluss

1) Thoma, Arch. f. patholog. Anatom. Bd. 93--113. 1883-1888. -v. Graefe's Archiv f. Ophthalmologie. Bd. 35. 1889. - Arch. f. path. Anat. Bd.116. 1889. - Beitr. z. path. Anat. u. allg. Path. Bd.10. 1891. - Untersuchungen über die Histogenese und Histomechanik des Gefässsystems. Stuttgart 1893. - Ueber die Erkrankungen der Gefässwandungen als Ursachen und als Folgen von Circulationsstörungen. Vortrag in der gemeinsamen Sitzung aller medicinischen Abtheilungen der Gesellschaft deutscher Naturforscher und Aerzte. Versammlung zu Düsseldorf 1898. 
Thoma, Ueber die Entstehung der falschen Knoten der Nabelschnur. 51 auf die Drehungen des Fötus unter normalen und unter pathologischen Bedingungen ausüben.

Jede Störung dieser Drehungsbewegungen, wio sie durch Umschlingungen der Nabelschnur und andere Umstände besonderer Art hervorgerufen werden, muss dann bei längerer Dauer zu Torsionsanomalien führen. Unter diesen aber gewähren die falschen Knoten der Nabelschnur die Anhaltspunkte, welche das gewichtigste Beweismaterial für diese Anschauungen liefern. Ihr anatomischer Bau spricht es klar und deutlich aus, dass sie entstehen durch ein gewaltsames Zurückdrehen der bereits gebildeten Spiralwindungen der Nabelschnurgefässe, also durch eine, in Folge besonderer Umstände eintretende Umkehr der bevorzugten Drehungsrichtung des Fötus.

Zugleich haben sich auch die ersten Anhaltspunkte ergeben zu dem Beweis der Hypothese, der zu Folge die umschriebenen, bulbösen und varicösen Erweiterungen der Arterien und Venen des Nabelstranges und der Placenta ihre Entstehung verdanken umschriebenen Ueberdehnungen und mikroskopischen Zerreissungen der Gewebselemente der Gefässwandungen, welche namentlich in Begleitung von Torsionsanomalien leicht eintreten. 